\title{
EFEITOS DO USO DIAGNÓSTICO E INTERATIVO DE MEDIDAS DE AVALIAÇÃO DE DESEMPENHO NA AMBIGUIDADE DE PAPÉIS E NO EMPODERAMENTO PSICOLÓGICO*
}

\author{
Ilse Maria Beuren** \\ Marcielle Anzilago ****

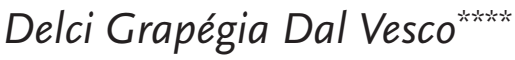

\footnotetext{
* doi: 10.11144/Javeriana.cao.31-56.ucdim. El artículo se recibió el 08/02/2018 y se aprobó 15/05/2018. Sugerencia de citación: Beuren, I., Anzilago, M., \& Dal Vesco, D. G. (2018). Efeitos do uso diagnóstico e interativo de medidas de avaliação de desempenho na ambiguidade de papéis e no empoderamento psicológico. Cuadernos de Administración, 31(56), 55-80. http://dx.doi.org/10.11144/Javeriana.cao.3156.ucdim

** Universidade Federal de Santa Catarina, Florianópolis, Brasil. E-mail: jlklein@uol.com.br

*** Universidade Federal de Santa Catarina, Florianópolis, Brasil. E-mail: marcianzilago@gmail.com

**** Universidade Estadual do Oeste do Paraná, UNIOESTE de Cascavel, Paraná, Brasil. E-mail: delcigrape@ gmail.com
} 
Efeitos do uso diagnóstico e interativo de medidas de avaliação de desempenho na ambiguidade de papéis e no empoderamento psicológico

Efectos del uso diagnóstico e interactivo de medidas de evaluación de desempeño en la ambigüedad de los papeles $\mathrm{y}$ en el empoderamiento psicológico

\section{Effects of the diagnostic and interactive use of performance assessment measures in role ambiguity and psychological empowerment}

\section{REsUMO}

Este estudo analisa a associação do uso diagnóstico e interativo de medidas de avaliação de desempenho com a diminuição da ambiguidade de papéis e o aumento do empoderamento psicológico de gestores. Pesquisa de levantamento foi realizada em uma cooperativa agroindustrial, na qual 52 gestores responderam o questionário baseado no estudo de Marginson, McAulay, Roush e Van Zijl (2014). Os resultados da modelagem de equações estruturais mostram que o uso diagnóstico e interativo das medidas de avaliação de desempenho não está associado com empoderamento psicológico. Porém, se confirma que o desempenho gerencial dos gestores é afetado pela ambiguidade de papéis.

Palavras-chave: medidas de avaliação de desempenho, controle diagnóstico, controle interativo, ambiguidade de papéis, empoderamento psicológico. Códigos JEL: $\mathrm{M}_{14}, \mathrm{M}_{4} 1$

\section{RESUMEN}

Este estudio analiza la asociación del uso diagnóstico y interactivo de medidas de evaluación del desempeño con la disminución de la ambigüedad de papeles y el aumento de empoderamiento psicológico de los gestores. La encuesta se realizó en una cooperativa agroindustrial, en la que 52 gestores respondieron al cuestionario basado en el estudio de Marginson, McAulay, Roush y Van Zijl (2014). Los resultados del modelado de las ecuaciones estructurales muestran que el uso diagnóstico e interactivo de las medidas de evaluación del desempeño no está asociado con el empoderamiento psicológico. Sin embargo, se confirma que el desempeño gerencial de los gestores es afectado por la ambigüedad de papeles.

\section{Palabras clave: medidas de evaluación de desempeño, control diagnóstico, control interactivo, ambigüedad de papeles, empoderamiento psicológico. Códigos JEL: M14, M41}

\section{ABSTRACT}

This study analizes the association of the diagnostic and interactive use of performance assessment measures with the decreased role ambiguity and increased psychological empowerment of managers. Survey research was conducted in an agro-industrial cooperative, in which 52 managers answered the questionnaire based on the study of Marginson, McAulay, Roush and Van Zijl (2014). The results of modeling of structural equations show that the diagnostic and interactive use of the performance assessment measures is not associated with psychological empowerment. However, it is confirmed that managerial performance of the managers is affected by role ambiguity.

Keywords: performance assessment measures, diagnostic control, interactive control, role ambiguity, psychological empowerment. JEL Codes: M14, M41 


\section{Introdução}

0 envolvimento dos empregados com os valores e objetivos da organização é essencial para resultados positivos no trabalho, como a satisfação no trabalho e a melhoria do desempenho. Isto obriga as empresas a revisar suas estratégias relativas à posição e processos necessários para fornecer sistemas com capacidade de gestão mais dinâmica (Neely, 2005). Gomes, Yasin e Lisboa (2004) afirmam que haverá impacto positivo no desempenho da organização se o sistema de gestão de desempenho é projetado ou redesenhado de forma adequada. No entanto, Lima, Costa e Angelis (2008) sugerem que não há nexo de causalidade entre essas iniciativas de redesenho e melhorias gerais no desempenho.

Compreender o papel de um sistema de medição de desempenho é o primeiro passo na definição das capacidades e funções que irão apoiar a gestão estratégica (Lima, Costa e Angelis, 2008). Tan e Platts (2009) destacam a importância de estabelecer relações causais entre a estratégia de negócio e o desenho do sistema de medição de desempenho. Sugerem ainda que há construções teóricas que medeiam a relação entre a estratégia e medidas de desempenho. Estas ligações devem ser estudadas em suas características estruturais e dinâmicas em diferentes ambientes organizacionais.

Marginson et al. (2014) analisaram as consequências psicológicas do uso dos sistemas de controle diagnóstico e interativo na avaliação de desempenho em uma empresa do setor de telecomunicações NewCom, na Nova Zelândia. Os autores constataram em seu estudo que o uso dos sistemas de avaliação de desempenho pode gerar efeitos positivos, elevando o nível do desempenho gerencial. Em termos gerais, concluíram que os sistemas de avaliação de desempenho tendem a diminuir os efeitos da ambiguidade de papéis, enquanto que aumentam os efeitos do empoderamento psicológico de gestores.

Nesta perspectiva, a motivação central da presente pesquisa é, por um lado, ressaltar a importância dos impactos psicológicos que podem ser produzidos pelo uso diagnóstico e interativo de medidas de avaliação de desempenho, afetando positiva ou negativamente a atividade dos gestores. $\mathrm{E}$, por outro, apontar que o uso dessas medidas de avaliação de desempenho pode sofrer variações nos seus efeitos psicológicos, dependendo dos princípios organizacionais onde são implementados. Objetiva-se verificar a associação do uso diagnóstico e interativo de medidas de avaliação de desempenho com a diminuição da ambiguidade de papéis e o aumento do empoderamento psicológico de gestores. 
Em primeiro lugar, a avaliação de desempenho dos gestores pode resultar em percepções injustas se forem atribuídas aos gestores responsabilidades que não fazem parte do seu papel. 0 não estabelecimento da função e autoridade de um indivíduo na organização pode desencadear conflito e ambiguidade de papéis (Katz e Kanh, 1978). 0 termo ambiguidade de papéis refere-se a essas incertezas sobre o papel de um indivíduo na empresa (Kahn, 1973). Por um lado, o conflito provém da incompatibilidade de tarefas ou quando as cobranças não se enquadram nos valores de quem as exerce. Por outro, a ambiguidade decorre da falta de informações ou autoridade para a realização das atividades (McNally, 1980).

Em segundo lugar, o acesso à qualidade da informação está positivamente associado com empoderamento (empowerment) psicológico, que é um construto motivacional que se distingue da simples delegação de poder de decisão (Spreitzer, 1995). Pelo contrário, a falta de informações sobre o desempenho pode ter efeitos adversos na capacitação. Ampla literatura sustenta esta ligação entre informações de desempenho e motivação, que é fundamental ao desenvolvimento da capacitação (Chiles e Zorn, 1995; Quinn e Spreitzer, 1997). Nesse sentido, é importante que as avaliações de desempenho tenham suporte em teorias psicológicas que indiquem quais mecanismos cognitivos e motivacionais são suscetíveis de explicar a relação entre as suas medidas (Collins, 1982).

Em terceiro lugar, indivíduos capacitados possuem melhor desempenho em comparação aos menos capacitados (Liden, Wayne e Sparrowe, 2000). Decorre que o empoderamento aumenta tanto a iniciativa e a persistência quanto a influência no comportamento nas tarefas dos gestores. Em níveis mais elevados de capacidade psicológica, o empoderamento leva o esforço e sua intensidade a um nível maior, gerando persistência e flexibilidade (Conger e Kanungo, 1988; Thomas e Velthouse, 1990). Cada dimensão do empoderamento está diretamente relacionada a comportamentos, com reflexos no desempenho. Isso porque os gestores atribuem mais significado às suas tarefas ou se preocupam mais em relação ao seu trabalho. Isto leva a um maior comprometimento e esforço em suas tarefas, inclusive persistindo essa posição face aos obstáculos (Thomas e Velthouse, 1990; Liden, Wayne e Sparrowe, 2000).

Neste sentido, a questão norteadora desta pesquisa é: Quais os efeitos do uso diagnóstico e interativo de medidas de avaliação de desempenho com ambiguidade de papéis e empoderamento psicológico de gestores? No presente estudo aplicaram-se os instrumentos e a metodologia de Marginson et al. (2014) para estimar os efeitos do uso diagnóstico e interativo de medidas de avaliação de desempenho em uma cooperativa 
agroindustrial com unidades estabelecidas em diversas regiões do Brasil. Contrário aos resultados de Marguson et al. (2014), os achados não mostram que o empoderamento psicológico esteja necessariamente associado ao uso diagnóstico e interativo de medidas de avaliação de desempenho. Porém, se confirma que a ambiguidade de papéis tem um efeito negativo no desempenho gerencial.

Os resultados divergentes em relação ao estudo base podem decorrer de princípios organizacionais distintos, como é o caso de cooperativas, com efeitos psicológicos diferentes nas medidas de avaliação de desempenho. Assim, este estudo denota relevância pela escassa literatura sobre o tema, especialmente em periódicos nacionais da área contábil. Embora se tenha identificado alguns estudos de contabilidade gerencial no Brasil, como os de Klann e Beuren (2014) e Nascimento e Beuren (2014), que tangenciam o tema, ele ainda é pouco pesquisado se comparado com publicações em periódicos estrangeiros, em que se identificaram mais pesquisas, como as de Conger e Kanungo (1988), Thomas e Velthouse (1990), Manz e Sims (1993), Spreitzer (1995), Hechanova, Alampay e Franco (2009).

\section{Referencial teórico e hipóteses}

\section{Sistemas de Controle Gerencial e Medidas de Avaliação de Desempenho}

Os sistemas de controle gerencial estão relacionados diretamente com a operacionalização das estratégias organizacionais. Eles têm como finalidade alertar os gestores para as incertezas estratégicas, minimizar problemas e otimizar as oportunidades (Anthony e Govindarajan, 2008). Simons (1995) propôs um modelo de sistema de controle gerencial com foco no crescimento empresarial ao gerar motivação a partir do compartilhamento de informações e do aprendizado organizacional. Este modelo compõe-se de quatro alavancas de controle, denominadas de: sistemas de crenças, sistemas de limites, sistemas de controle interativo e sistemas de controle diagnóstico.

No presente estudo interessam em específico as duas últimas alavancas de controle. 0 controle interativo objetiva monitorar incertezas estratégicas ou ambientais, promovendo o aprendizado e potencializando as estratégias emergentes (Simons, 1995). 0 controle interativo está orientado a facilitar a formação de um clima de aprendizado contínuo (Henri, 2006; Anthony e Govindarajan, 2008). 0 sistema de controle interativo estimula a pesquisa e aprendizagem por meio de novas estratégias, instigando 
os membros da organização frente às oportunidades e ameaças do ambiente (Simons, 1995; Henri, 2006).

0 controle diagnóstico é considerado o principal foco dos sistemas de controle gerencial tradicionais, os quais são idealizados para assegurar o cumprimento das metas. Porém, os gestores tendem a ter pouca preocupação com as mesmas. 0s sistemas de controle diagnóstico podem ser usados em organizações de qualquer tamanho, em que a complexidade das operações e o número das decisões tomadas pelos subordinados considerem os parâmetros predefinidos pelos gestores. É preciso que os gestores tenham garantias de que estas decisões estejam em consonância com os objetivos organizacionais.

Assim, o controle interativo é utilizado para nortear as estratégias organizacionais, enquanto o controle diagnóstico tem a função de assegurar a realização das metas. No modelo proposto por Simons (1995), todas as partes da organização podem agir de forma proativa, assim como aproveitar as oportunidades inesperadas ao lidar com os problemas. Dessa forma, as ações serão estrategicamente importantes, de modo que as experiências bem-sucedidas sejam repetidas e ampliadas com o passar do tempo. A organização ajustará suas estratégias para capitalizar a aprendizagem gerada pelas novas ideias de seus membros (Simons, 1995).

Depreende-se que há relação entre sistemas de controle gerencial e medidas de avaliação de desempenho. As medidas de avaliação de desempenho são usadas para quantificar a eficiência e a eficácia das ações, um parâmetro utilizado para quantificar a eficiência e/ ou eficácia das medidas anteriores em comparação com o padrão estabelecido (Neely et al., 1995). Desse modo, a medição do desempenho é dependente das medidas utilizadas para capturar a essência do desempenho, bem como para a definição das metas (Gunasekaran, Patel e Mcgaughey, 2004).

\section{Ambiguidade de papéis, Empowerment psicológico e desempenho gerencial}

A ambiguidade de papéis refere-se ao nível de incerteza do indivíduo face às expectativas dos outros sobre o seu papel e o desempenho gerencial (Rizzo, House e Lirtzman, 1970; House e Rizzo, 1972; Churchill Jr., Ford e Walker Jr., 1976; Michaels, Ralph e Erich, 1987). A ambiguidade de papéis ocorre quando o indivíduo sente que não tem informação necessária para desempenhar seu papel adequadamente, ficando indeciso quanto ao modo de corresponder às expectativas (Kahn, Wolfe, Quinn e Snoek, 1964; 
Churchill Jr. et al., 1976). Assim, a pessoa incumbida de desempenha-la não é capaz de realiza-la conforme às expectativas.

0 empowerment ou empoderamento também se espera ter reflexos no desempenho gerencial e é definido por Conger e Kanungo (1988) como a motivação de autoeficácia. Esta implica a concessão de autoridade e autonomia necessárias aos subordinados para eles tomarem decisões no local de trabalho (Clark, Hartline e Jones, 2009). Tal exige que os gestores compartilhem informações e conhecimento, e assim melhorem o desempenho dos subordinados no trabalho (Ford e Fottler, 1995). Konczak, Stelly e Trusty (2000) destacam a necessidade de os líderes apoiar o desenvolvimento de habilidades e incentivar seus subordinados a participar na tomada de decisões. Nesse sentido, devem-se tratar os erros e contratempos como oportunidades de aprendizagem.

Há evidências na literatura de que o empoderamento psicológico está relacionado com resultados positivos nas atitudes dos empregados, tais como: comprometimento organizacional (Manz e Sims, 1993), comportamento inovador (Spreitzer, 1995), satisfação e desempenho no trabalho (Hechanova, Alampay e Franco, 2009). Nesta perspectiva, a capacitação deve refletir a orientação de um indivíduo para o seu papel de trabalho, manifestada em um conjunto de quatro cognições: avaliação, competência, autodeterminação e impacto (Thomas e Velthouse, 1990).

Para Spreitzer (1995), estas cognições se combinam aditivamente para produzir uma sensação geral de capacitação nos indivíduos. Juntas, refletem-se na orientação para o desempenho das atividades, ou seja, por uma orientação ativa, em que os desejos individuais são capazes de moldar o contexto do desempenho de suas atividades (Thomas e Velthouse, 1990; Spreitzer, 1995). As quatro dimensões formam uma construção geral de capacitação psicológica, ou seja, especificam um conjunto quase completo de cognições para o empoderamento psicológico.

Liden, Wayne e Sparrowe (2000) examinaram o efeito agência, que consiste nas diferentes dimensões da capacitação psicológica dos gestores. Os autores descobriram que a dimensão do significado de empoderamento psicológico está associada com a função de agência, essa aferida pela relação da característica do trabalho e da satisfação no trabalho com o comprometimento organizacional. Assim, o empoderamento psicológico é uma construção que aumenta as experiências difíceis e desafiadoras dos gestores. Desse modo, pode-se supor que a capacitação irá desempenhar um papel moderador entre confiança organizacional e engajamento no trabalho (Spreitzer, 1995). 
Seibert, Wang e Courtright (2011) constataram em uma meta-análise que a liderança tem efeitos mais fortes na capacitação psicológica. Observaram ainda um efeito positivo para as relações entre capacitação psicológica, execução de tarefas e comportamento de cidadania organizacional. A partir desses resultados, os autores inferiram que a capacitação psicológica representa um mecanismo de compreensão dos efeitos motivacionais de autoeficácia dos líderes transformacionais, assim como das suas ligações com os subordinados no desempenho do trabalho e seu comportamento.

A relação positiva entre desempenho gerencial e empoderamento psicológico indica que quanto maior a motivação do gestor e a percepção sobre sua influência no desempenho da organização, maior será seu desempenho gerencial. De igual modo, esta relação positiva permite prever uma maior eficiência dos gestores, derivada do fato deles acreditar que a sua atuação pode contribuir para o desempenho organizacional (Hall, 2008). Segundo o modelo, os gestores que outorgam um maior significado de seu trabalho tendem a se esforçar mais, serem mais comprometidos com suas tarefas e mais persistentes diante dos obstáculos, resultando em maior esforço e iniciativa na organização (Thomas e Velthouse, 1990).

\section{Estudo de Marginson et al. (2014) e Hipóteses}

Marginson et al. (2014) realizaram uma pesquisa com gestores da NewCom, maior empresa mundial do setor de telecomunicações da Nova Zelândia. 0 estudo enfatiza a relação entre as variáveis do empowerment e o desempenho não-financeiro, e as medidas estratégicas de apoio e contínua adaptação ao contexto de concorrência. 0s autores objetivaram verificar as consequências psicológicas do uso dos sistemas de controle diagnóstico e interativo nas medidas de avaliação de desempenho.

As variáveis sistemas de controle interativo (medidas de avaliação de desempenho não-financeiras) e sistemas de controle diagnóstico (medidas de avaliação de desempenho não-financeiras) foram utilizadas por Marginson et al. (2014) sob o argumento de que elas são cada vez mais reconhecidas na literatura como fornecedoras de uma estrutura adequada para o entendimento das consequências da avaliação de desempenho. Nas hipóteses, focalizaram o comportamento dos gestores em relação à ambiguidade de papéis, ao empowerment psicológico e ao uso interativo e diagnóstico das medidas de avaliação de desempenho. 
Com o intuito de contribuir para o avanço do tema, no presente estudo alterou-se o campo da pesquisa para uma organização cooperativa, no pressuposto de que princípios organizacionais distintos podem ter efeitos psicológicos diferentes nas medidas de avaliação de desempenho. Nesse sentido, as hipóteses elaboradas no estudo de Marginson et al. (2014) foram mantidas, descritas a seguir e representadas na Figura 1:

H1: 0 uso diagnóstico de medidas de avaliação de desempenho está associado negativamente com a ambiguidade de papéis.

H2: 0 uso interativo de medidas de avaliação de desempenho está associado positivamente com o empowerment psicológico.

H3: A ambiguidade de papéis está associada negativamente com o empowerment psicológico.

H4a: A ambiguidade de papéis está associada negativamente com o desempenho gerencial. H4b: 0 empowerment psicológico está associado positivamente com o desempenho gerencial.

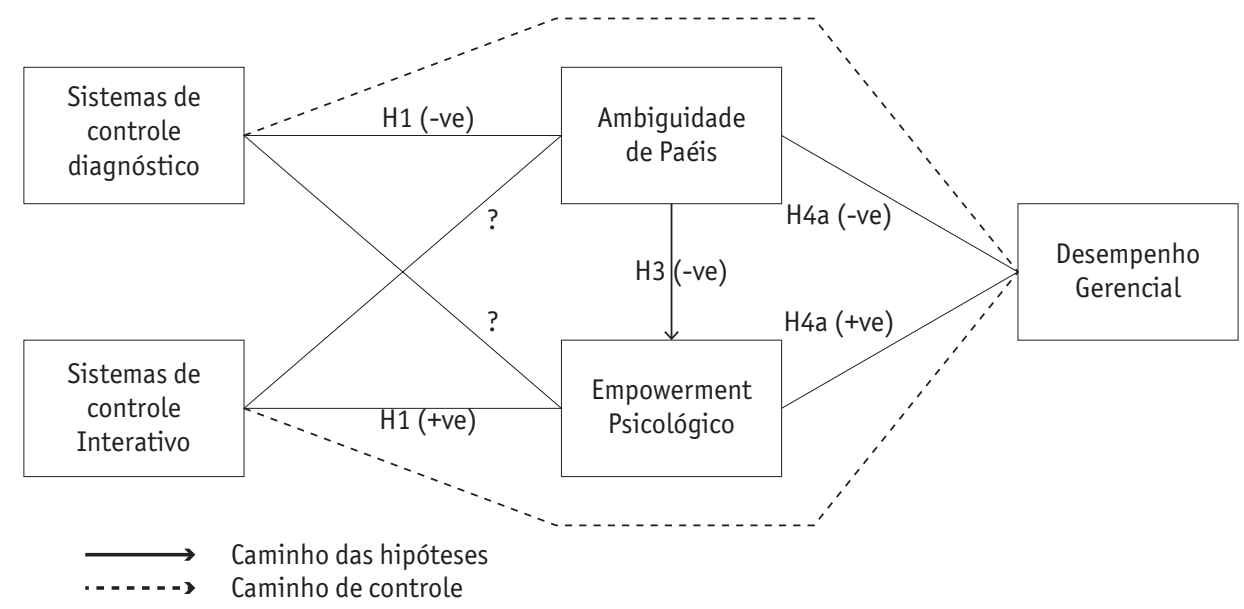

Figura 1. Modelo teórico

Fonte: Marginson et al. (2014, p. 68).

Na análise dos resultados, Marginson et al. (2014) constataram que ambiguidade de papéis está associada negativamente com uso diagnóstico e uso interativo de medidas 
de avaliação de desempenho. Portanto, o estudo apoia a posição de que as medidas de avaliação de desempenho, quer de uso diagnóstico ou interativo, são fontes eficazes de informação para neutralizar a ambiguidade de papéis. Além disso, as medidas não-financeiras de avaliação de desempenho estimulam o debate sobre se elas são fonte de informações necessárias ao empoderamento psicológico. Decorre que a eficácia das medidas de avaliação de desempenho também depende de como os sistemas de medição de desempenho são utilizados.

Os resultados do estudo de Marginson et al. (2014) sugerem que as metas organizacionais são esclarecidas pela participação dos subordinados e de interações interpessoais, em consonância com o previsto no sistema de controle interativo. Deste modo são incentivadas discussões entre os gestores, como um desafio de encorajamento e discussãodos pressupostos subjacentes. Igualmente, tende-se a promover maior consciência da capacidade do gestor atender suas metas individuais, melhorar sua capacidade de identificar-se com as metas corporativas e, consequentemente, envolver todos no estabelecimento das metas e objetivos da organização. A oportunidade para a discussão e o debate é provável que aconteça no uso de medidas não financeiras.

\section{Metodologia da pesquisa}

Esta pesquisa foi realizada por meio de um levantamento em uma das maiores cooperativas agroindustriais do Brasil, com diversas unidades estabelecidas nas regiões Sul e Centro-0este. A seleção dessa cooperativa deu-se em função de sua estrutura de gestão descentralizada de negócios e do contingente de gestores dos diversos níveis hierárquicos, aspectos fundamentais para averiguar se os efeitos psicológicos nas medidas de avaliação de desempenho diferem no caso de uma organização com princípios cooperativos.

A percepção dos gestores foi aferida por meio do instrumento de pesquisa traduzido do estudo de Marginson et al. (2014), composto dos construtos e das 30 assertivas, em escala Likert de sete pontos (Tabela 1). Para se assegurar da tradução adequada do questionário, fez-se a sua reversão para a língua inglesa. Também realizou-se um teste piloto com dois alunos de mestrado e dois pesquisadores, sendo que as alterações recomendas por ambos foram relativas a redação das assertivas. 


\section{Tabela 1}

\section{Construtos da pesquisa}

\begin{tabular}{|c|c|c|c|}
\hline Construtos & Elementos & Assertivas do questionário & Referências \\
\hline $\begin{array}{l}\text { Uso } \\
\text { diagnóstico } \\
\text { (UD) }\end{array}$ & $\begin{array}{l}\text { Medidas de } \\
\text { avaliação de } \\
\text { desempenho } \\
\text { financeiras }\end{array}$ & $\begin{array}{l}\text { 1. Acompanha o progresso em direção às metas. } \\
\text { 2. Monitora os resultados. } \\
\text { 3. Compara os resultados com as expectativas. } \\
\text { 4. Revê as principais metas. }\end{array}$ & $\begin{array}{l}\text { Vandenbosch } \\
\text { (1999) } \\
\text { Henri (2006) }\end{array}$ \\
\hline $\begin{array}{l}\text { Uso } \\
\text { interativo } \\
\text { (UI) }\end{array}$ & $\begin{array}{l}\text { Medidas de } \\
\text { avaliação de } \\
\text { desempenho não- } \\
\text { financeiras }\end{array}$ & $\begin{array}{l}\text { 5. Incentiva debates/discussões em reuniões. } \\
\text { 6. Incentiva contínuo desafio e debate sobre dados } \\
\text { subjacentes, suposições e planos de ação. } \\
\text { 7. Fornece uma visão comum da organização. } \\
\text { 8. Amarra as partes da organização no todo. } \\
\text { 9. Habilita sua área para se concentrar em problemas } \\
\text { comuns. } \\
\text { 10. Desenvolve um vocabulário comum na sua área. } \\
\text { 11. Habilita sua área para se concentrar em fatores } \\
\text { críticos de sucesso. }\end{array}$ & $\begin{array}{l}\text { Vandenbosch } \\
(1999) \\
\text { Henri (2006) }\end{array}$ \\
\hline
\end{tabular}

12. Divido meu tempo corretamente.

13. Tenho objetivos claros e planejados para o meu trabalho.

Ambiguidade Autoridade, de papéis Clareza e (AP) Confiança no superior.
14. Eu sei quais são minhas responsabilidades.

15. Tenho certeza de quanta autoridade possuo.

16. Eu sei exatamente o que é esperado de mim.

17. É claro para mim o que tem de ser feito.

18. Meu impacto sobre o que acontece em minha unidade é grande.

Impacto 19. Eu tenho pouco controle sobre o que acontece na minha unidade. *

20. Tenho uma influência significativa sobre o que acontece na minha unidade.

21. Estou confiante sobre a minha capacidade de fazer 0 meu trabalho.

22. Estou certo de mim mesmo sobre minhas capacidades para as atividades do meu trabalho.

House e Rizzo

23. 0 trabalho que faço é significativo para mim.

Significado

24. Minhas atividades do trabalho são pessoalmente significativas para mim.

25. 0 trabalho que eu faço é sem importância para mim. * $\begin{array}{ll}\text { Autodeterminação } & \text { 26. Tenho autonomia } \\ \text { faço meu trabalho. }\end{array}$
Spreitzer

(1995)

(EP) 


\begin{tabular}{llll}
\hline Construtos & Elementos & \multicolumn{1}{c}{ Assertivas do questionário } & Referências \\
\hline & & $\begin{array}{l}\text { 27. Eu posso decidir por conta própria como fazer o meu } \\
\text { trabalho. }\end{array}$ & \\
& & $\begin{array}{l}\text { 28. Tenho poucas oportunidades de independência e } \\
\text { liberdade em como fazer o meu trabalho. * }\end{array}$ & \\
& & 29. Indique a classificação de seu desempenho recente. & Mahoney et \\
$\begin{array}{l}\text { Desempenho } \\
\text { gerencial } \\
\text { (DG) }\end{array}$ & $\begin{array}{l}\text { Avaliação, Super- } \\
\text { visão, Negociação } \\
\text { e Nível hierárquico }\end{array}$ & $\begin{array}{l}\text { 30. Como você se avalia no cargo de gerente em } \\
\text { comparação com outros gerentes de mesmo nível, } \\
\text { levando tudo em consideração? }\end{array}$ & al. (1965) \\
\hline
\end{tabular}

Nota: * As assertivas 19, 25 e 28 estão com escala reversa.

Fonte: elaboração própria com base em Marginson et al. (2014).

Em relação aos procedimentos de coleta de dados, o contato com a cooperativa deuse por meio do envio de uma carta de apresentação direcionada ao Departamento de Recursos Humanos, em que foram expostos os objetivos da pesquisa. 0 questionário foi direcionado aos gestores da cooperativa dos diversos níveis hierárquicos. 0 questionário foi enviado pelo Google Docs aos 112 gestores da cooperativa, no período de maio a junho de 2014, e obteve-se o retorno de um total de 52 questionários válidos.

Na análise dos dados utilizou-se a modelagem de equações estruturais (SEM). 0 Partial Least Squares-Structural Equation Modeling (PLS-SEM) é uma técnica de estimação de regressão linear, que se baseia na decomposição de matrizes de variáveis e de covariáveis (Bido, Silva, Souza e Godoy, 2010). Na análise efetuou-se testes de validação e adequação do modelo, executados no software SmartPLS.

0 uso do PLS é pertinente ao presente estudo pois permite trabalhar com amostras pequenas e é capaz de lidar com alta complexidade. Esta técnica baseia-se no estudo de um sistema de relações lineares entre variáveis latentes que é resolvido por partes, uma de cada vez. 0 principal objetivo do PLS é estimar a variância de construtos endógenos e suas respectivas variáveis manifestas, a um nível de significância de 0,05 (Bido et al., 2010). 0 PLS é particularmente útil neste estudo, já que a técnica testa hipóteses com dados mínimos e é robusta para amostras pequenas (Hair Jr., Black, Babin, Anderson e Tatham, 2009).

\section{Descrição e análise dos resultados}

\section{Análises descritivas}

Na Tabela 2 evidencia-se os resultados das estatísticas descritivas, em específico de medidas de mínimo, máximo, média, mediana e desvio padrão das respostas ao questionário. 


\section{Tabela 2}

Estatística descritiva

\begin{tabular}{lccccc}
\hline \multicolumn{1}{c}{ Construtos } & Mínimo & Máximo & Média & Mediana & Desvio Padrão \\
\hline Ambiguidade de Papéis (AP) & 2 & 7 & 6,04 & 6 & 0,805 \\
Empowerment Psicológico (EP) & 1 & 7 & 5,48 & 6 & 1,385 \\
Desempenho Gerencial (DG) & 1 & 7 & 5,87 & 6 & 0,998 \\
Uso Diagnóstico (UD) & 3 & 5 & 4,55 & 5 & 0,555 \\
Uso Interativo (UI) & 1 & 5 & 4,31 & 4 & 0,746 \\
\hline
\end{tabular}

Fonte: Dados da pesquisa.

Observa-se na Tabela 2, que a ambiguidade de papéis se apresenta elevada, com média 6,04, o que denota que não há clareza nas informações e especificações para a execução das tarefas (Katz e Kanh, 1978). 0 empowerment psicológico também foi alto, com média 5,48 , mas com desvio padrão maior do que em ambiguidade de papéis. No desempenho gerencial, verifica-se um elevado nível de envolvimento dos gestores com as atividades de planejamento, avaliação, supervisão e negociação. Nas medidas de avaliação de desempenho, tem-se que os gestores possuem comprometimento com os sistemas de controle idealizados para assegurar a realização das metas previstas.

\section{Avaliação do Modelo de Mensuração e Estrutural}

Para testar a validade dos indicadores de medição dos construtos do modelo efetuou-se a análise das cargas fatoriais por meio da matriz cross loadings. Na Tabela 3 são apresentadas as cargas fatoriais de cada um dos indicadores finais dos construtos.

\section{Tabela 3}

Matriz Cross Loadings - cargas fatoriais finais

\begin{tabular}{lccccc}
\hline \multicolumn{1}{c}{ Indicadores } & AP & DG & EP & UD & UI \\
\hline AP12. Divido meu tempo corretamente & $\mathbf{0 , 7 5 2}$ & $\mathbf{0 , 0 2 0}$ & $\mathbf{0 , 4 2 1}$ & $-0,449$ & $-0,020$ \\
AP13. Tenho objetivos claros e planejados para o meu trabalho & $\mathbf{0 , 8 9 2}$ & $\mathbf{0 , 1 6 0}$ & $\mathbf{0 , 4 3 3}$ & $-0,442$ & $-0,053$ \\
AP14. Eu sei quais são minhas responsabilidades & $\mathbf{0 , 6 9 6}$ & $\mathbf{0 , 3 2 0}$ & $\mathbf{0 , 3 9 5}$ & $-0,427$ & $-0,265$ \\
AP15. Tenho certeza de quanta autoridade possuo & $\mathbf{0 , 7 3 0}$ & $\mathbf{0 , 0 8 6}$ & $\mathbf{0 , 5 0 0}$ & $-0,480$ & $-0,234$ \\
AP16. Eu sei exatamente o que é esperado de mim & $\mathbf{0 , 9 0 8}$ & $\mathbf{0 , 2 0 1}$ & $\mathbf{0 , 5 3 1}$ & $-0,502$ & $-0,084$ \\
AP17. É claro para mim o que tem de ser feito & $\mathbf{0 , 7 3 2}$ & $\mathbf{0 , 0 4 0}$ & $\mathbf{0 , 4 0 6}$ & $-0,367$ & 0,003 \\
DG29. Indique a classificação de seu desempenho recente & $\mathbf{0 , 2 1 8}$ & $\mathbf{0 , 8 9 1}$ & $\mathbf{0 , 4 2 9}$ & $-\mathbf{0 , 0 8 4}$ & $-0,248$ \\
\hline
\end{tabular}




\begin{tabular}{|c|c|c|c|c|c|}
\hline Indicadores & AP & DG & EP & UD & UI \\
\hline $\begin{array}{l}\text { DG30. Como você se avalia no cargo de gerente em } \\
\text { comparação com outros gerentes de mesmo nível, levando } \\
\text { tudo em consideração? }\end{array}$ & 0,066 & 0,808 & 0,309 & $-0,027$ & $-0,289$ \\
\hline $\begin{array}{l}\text { EP26. Tenho autonomia significativa para determinar como } \\
\text { faço meu trabalho }\end{array}$ & 0,569 & 0,337 & 0,800 & $-0,418$ & $-0,258$ \\
\hline $\begin{array}{l}\text { EP27. Eu posso decidir por conta própria como fazer o meu } \\
\text { trabalho }\end{array}$ & 0,403 & 0,219 & 0,710 & $-0,327$ & $-0,188$ \\
\hline $\begin{array}{l}\text { EP28. Tenho poucas oportunidades de independência e } \\
\text { liberdade em como fazer o meu trabalho }\end{array}$ & 0,155 & 0,410 & 0,610 & 0,127 & 0,004 \\
\hline UD1. Acompanha o progresso em direção às metas & $-0,524$ & $-0,087$ & $-0,348$ & 0,957 & 0,486 \\
\hline UD2. Monitora os resultados & $-0,561$ & $-0,046$ & $-0,324$ & 0,959 & 0,465 \\
\hline $\begin{array}{l}\text { UI6. Incentiva contínuo desafio e debate sobre dados } \\
\text { subjacentes, suposições e planos de ação }\end{array}$ & $-0,137$ & $-0,268$ & $-0,216$ & 0,392 & 0,819 \\
\hline UI7. Fornece uma visão comum da organização & $-0,126$ & $-0,176$ & $-0,162$ & 0,398 & 0,761 \\
\hline UI8. Amarra as partes da organização no todo & $-0,030$ & $-0,282$ & $-0,152$ & 0,350 & 0,710 \\
\hline
\end{tabular}

Fonte: Dados da pesquisa.

A Tabela 3 mostra que não apresentaram cargas fatoriais confirmatórias (CFA) satisfatórias: EP 18, 19, 20, 21, 22, 23, 24, 25 do constructo Empowerment Psicológico; UD 3 e 4 do constructo Uso Diagnóstico; e UI 5, 9, 10 e 11 do constructo Uso Interativo. Hair Jr. et al. (2009) advertem que estimativas de cargas podem ser estatisticamente significantes, mas pequenas demais para se qualificarem como bons itens (cargas padronizadas abaixo de 0,50 ) em CFA. Além disso, os autores afirmam que itens com cargas pequenas se tornam candidatos à eliminação caso demandem melhoria na validade convergente (AVE) ou na confiabilidade composta (CC). No caso dos indicadores mencionados, a AVE e a CC só atingiram valores aceitáveis quando da exclusão dos mesmos.

Assim, optou-se por excluir estes indicadores para uma melhor adequação ao modelo proposto. Após a eliminação, a matriz de cargas cruzadas das CFA indicou que apenas duas questões (AP14 e EP28) mostraram cargas inferiores a 0,70, mas superiores ao mínimo recomendado de 0,40, conforme Hair Jr. et al. (2009). No estudo de Marginson et al. (2014), diferentemente, foram eliminados somente os itens 8 e 9 do construto uso interativo, pois apresentavam carga fatorial abaixo de 0,50. Essas diferenças podem advir do campo de pesquisa distinto do estudo base, como da constituição jurídica, do setor econômico, do contexto social e econômico, aspectos a serem investigados em pesquisas futuras.

Na avaliação do modelo de mensuração inseriram-se variáveis de controle, em consonância com o modelo de Marginson et al. (2014), que aferiram três características: 
idade, sexo, escolaridade. Essas variáveis podem influenciar crenças gerenciais, valores, opiniões e ações dos indivíduos, consequentemente, se refletir nas respostas. Contudo, neste estudo, em função da homogeneidade da amostra a variável sexo foi desconsiderada, já que todos os respondentes são do gênero masculino. Também foi inserida a variável de controle nível hierárquico, sob o argumento de que a autoridade aumenta com o tempo de serviço, assim potencialmente influencia os sentimentos de poder (Marginson et al., 2014).

As variáveis de controle (idade, gênero, formação acadêmica e nível hierárquico) no artigo base não foram representadas graficamente no modelo, mas fazem parte do caminho de controle. 0 caminho de controle foi representado por linhas tracejadas e medido pelas variáveis de controle. As variáveis de controle, neste estudo, assim como no de Marginson et al. (2014), não foram estatisticamente significantes para nenhum construto ao $\mathrm{p}$-value de 0,05 , contudo modificam os resultados do modelo e, portanto, foram mantidas.

Para os testes de validação e adequação das variáveis latentes do modelo estrutural, procedeu-se à análise da confiabilidade composta, do alfa de Cronbach, da validade convergente (AVE) e da validade discriminante. A confiabilidade composta é empregada para avaliar se a amostra não possui vieses ou ainda se as respostas são confiáveis (Bido et al., 2008). 0 alfa de Cronbach é utilizado para medir a confiabilidade dos constructos (Hulland, 1999). A AVE refere-se à variância média extraída, que demonstra a variância compartilhada entre os indicadores de cada variável latente ou construto do modelo (Hair Jr. et al., 2009). Deve-se também analisar a confiabilidade discriminante. Na Tabela 4, apresentam-se os valores de AVE, confiabilidade composta e alfa de Cronbach para cada construto.

\section{Tabela 4}

\section{Confiabilidade Composta, Alfa de Cronbach e Validade Convergente}

\begin{tabular}{ccccc}
\hline Construtos & AVE & Confiabilidade composta & Alfa de Cronbach & Comunalidades \\
\hline AP & 0,623 & 0,907 & 0,876 & 0,643 \\
EP & 0,505 & 0,752 & 0,629 & 0,752 \\
DG & 0,723 & 0,839 & 0,623 & 0,650 \\
UD & 0,918 & 0,957 & 0,911 & 0,687 \\
UI & 0,585 & 0,808 & 0,657 & 0,684 \\
Controle & 0,508 & 0,326 & - & 0,489 \\
\hline
\end{tabular}

Fonte: Dados da pesquisa. 
Observa-se na Tabela 4 que todas as cargas para a AVE se mostraram estatisticamente significantes, isto é, com valores iguais ou superiores a 0,50 conforme preconiza Fornell e Larcker (1981). A confiabilidade composta, excluindo a variável de controle, também atendeu aos padrões considerados satisfatórios. 0 alfa de Cronbach excedeu a 0,70 para os constructos AP e UD, o que indica confiabilidade aceitável. Os demais construtos não atingiram esse valor, mas segundo Hair Jr. et al. (2009), o valor mínimo aceitável para o alfa é de 0,70, podendo diminuir para 0,60 em pesquisas exploratórias. Em qualquer caso, esses valores abaixo de 0,60 exigem cautela na análise dos resultados.

Dessa forma, a confiabilidade composta e o alfa de Cronbach asseguram que a amostra está livre de vieses e que o instrumento de coleta de dados é confiável. Para confirmar a validade discriminante das variáveis latentes do modelo determinou-se o valor da AVE. De acordo com Fornell e Larcker (1981), ela é confirmada quando o valor da raiz quadrada da AVE é maior que os valores absolutos das correlações com as demais variáveis latentes. Neste estudo testou-se a validade discriminante pelo critério de Fornell e Larcker (1981), cujos resultados são apresentados na Tabela 5.

\section{Tabela 5}

Validade discriminante

\begin{tabular}{ccccccc}
\hline Construtos & AP & DG & EP & UD & UI & Controle \\
\hline AP & $\mathbf{0 , 7 8 9}$ & - & - & - & - & - \\
DG & 0,177 & $\mathbf{0 , 8 5 9}$ & - & - & - & - \\
EP & 0,572 & 0,440 & $\mathbf{0 , 7 1 1}$ & - & - & - \\
UD & $-0,567$ & $-0,069$ & $-0,350$ & $\mathbf{0 , 9 5 8}$ & - & - \\
UI & $-0,139$ & $-0,311$ & $-0,236$ & 0,496 & $\mathbf{0 , 7 6 5}$ & - \\
Controle & $-0,259$ & $-0,115$ & 0,127 & $-0,146$ & 0,119 & $\mathbf{0 , 6 6 5}$ \\
\hline
\end{tabular}

Fonte: Dados da pesquisa.

Verifica-se na validade discriminante apresentada na Tabela 5, que nenhuma das correlações entre os construtos foi superior a raiz quadrada da AVE, indicando que existe validade discriminante e convergente no modelo proposto. No estudo de Marginson et al. (2014), a validade discriminante foi considerada satisfatória, exceto na variável AP, com carga de 0,48 , e o modelo apresentou validade convergente, com exceção da variável DG. Assim, depreende-se que o modelo de mensuração possui validade convergente e validade discriminante satisfatórios. 
Após a avaliação do modelo de mensuração, estimou-se o modelo estrutural. 0s coeficientes de caminho representam a força e a direção das relações entre as variáveis latentes e podem ser interpretados como coeficientes beta padronizados de regressões de mínimos quadrados comuns (Henseler, Ringle e Sinkovics, 2009). Para obter os erros padrão dos coeficientes de caminho utilizou-se o procedimento de bootstrapping (Davison e Hinkley, 2003). Ao dividir o coeficiente de caminho pelo erro padrão obtido pelo bootstraping, tem-se o valor t empírico, o qual permite uma avaliação do significado do coeficiente de trajetória correspondente (Chin, 1998). No procedimento bootstraping fizeram-se 2.000 substituições para estimar a significância estatística dos parâmetros. Complementou-se a análise calculando os valores de $\mathrm{R}^{2}$, que segundo Cohen (1988), representam a quantidade de variância em uma variável endógena explicada pelas variáveis exógenas.

0 bootstrapping não apresentou multicolinariedade no modelo. Alta correlação foi observada entre as variáveis UD->AP $(0,600)$ em comparação com as demais variáveis, $A P->D G(0,374), A P->E P(0,141), \operatorname{EP}->D G(0,368), U D->D G(0,111), U D->E P(0,028), U I-$ $>A P(0,146), U I->D G(0,442)$ e UI->EP $(0,136)$. $E$, as variáveis AP, DG e UD apresentaram cargas fatoriais altas, respectivamente $0,726,0,829$ e 0,954. Em consonância com a avaliação do modelo estrutural, na Tabela 6 demonstram-se as relações ou caminhos (path), que segundo Hair Jr. et al. (2009) apresenta a relação estabelecida entre dois construtos e sua significância.

\section{Tabela 6}

Efeitos da relação entre os construtos

\begin{tabular}{|c|c|c|c|c|c|c|c|}
\hline \multirow{2}{*}{$\begin{array}{l}\text { Relação entre os } \\
\text { construtos }\end{array}$} & \multirow{2}{*}{ Hipótese } & \multicolumn{3}{|c|}{ Efeito direto } & \multicolumn{3}{|c|}{ Efeito indireto } \\
\hline & & Valor & t-value & $p$-value & Valor & t-valor & $\mathrm{p}$-value \\
\hline$A P->D G$ & $\mathrm{H} 4 \mathrm{a}$ & $-0,111$ & 0,459 & $0,090 * * *$ & 0,297 & 1,968 & 0,050 \\
\hline$A P->E P$ & $\mathrm{H} 3$ & 0,591 & 4,118 & 0,281 & & & \\
\hline$E P->D G$ & $\mathrm{H} 4 \mathrm{~b}$ & 0,504 & 2,622 & 0,009 ** & & & \\
\hline UD->AP & $\mathrm{H} 1$ & $-0,661$ & 3,041 & $0,002^{*}$ & & & \\
\hline UD->DG & Path Controle & 0,111 & 0,600 & 0,549 & $-0,266$ & 1,260 & 0,208 \\
\hline UD->EP & $\mathrm{H} 1$ & 0,081 & 0,440 & 0,660 & $-0,390$ & 2,308 & 0,021 \\
\hline UI->AP & $\mathrm{H} 2$ & 0,189 & 1,145 & 0,253 & & & \\
\hline UI->DG & Path Controle & $-0,194$ & 2,865 & $0,004^{*}$ & 0,112 & 0,741 & 0,459 \\
\hline UI->EP & $\mathrm{H} 2$ & 0,136 & 1,255 & 0,210 & 0,094 & 1,186 & 0,236 \\
\hline$V C->A P$ & V.C. & $-0,232$ & 0,997 & 0,319 & $-0,107$ & 0,752 & 0,452 \\
\hline
\end{tabular}




\begin{tabular}{cccccccc}
\hline \multirow{2}{*}{$\begin{array}{c}\text { Relação entre os } \\
\text { construtos }\end{array}$} & Hipótese & \multicolumn{3}{c}{ Efeito direto } & \multicolumn{3}{c}{ Efeito indireto } \\
\cline { 3 - 7 } & & Valor & t-value & p-value & Valor & t-valor & p-value \\
\hline VC->DG & V.C. & $-0,216$ & 0,950 & 0,343 & 0,069 & 0,367 & 0,714 \\
VC->EP & V.C. & $-0,023$ & 0,090 & 0,928 & 0,070 & 0,529 & 0,597 \\
VC->UD & V.C. & $-0,146$ & 0,650 & 0,516 & & & \\
VC->UI & V.C. & 0,134 & 0,579 & 0,563 & & & \\
$R^{2}$ & AP: 0,348 & DG: 0,202 & EP: 0,355 & UD: 0,352 & UI: 0,214 & & \\
\hline
\end{tabular}

Obs.: Significante ao nível de ${ }^{*} 0,01 ;{ }^{* *} 0,05,{ }^{* *} 0,10$.

Fonte: Dados da pesquisa.

Nota-se na Tabela 6, que os efeitos diretos entre os construtos foram significativos para três hipóteses: H1 (UD->AP), H4a (AP->DG) e H4b (EP->DG). Além disso, foi significante para um caminho de controle (UI->AP), já para os demais caminhos não se apresentaram significantes no modelo proposto. As variáveis de controle não obtiveram significância, portanto, esses resultados precisam ser analisados com cautela devido à pesquisa ter sido realizada em uma cooperativa, com características diferentes de outras empresas, seja pelo ambiente, a cultura e aspectos de gestão. Nota-se que AP e EP possuem efeitos diretos positivos e significativos sobre DG, mas não sobre as demais variáveis, o que indica mediação. A variável UD demonstrou relação negativa direta e significativa com AP. Para os efeitos indiretos, somente a variável AP apresentou relação significativa e positiva com DG.

Pelo $\mathrm{R}^{2}$ nota-se ajustamento do modelo estatístico, em consonância com o preconizado por Hair Jr. et al. (2009). A ambiguidade de papéis (AP) explica 34,8\% dos valores observados no modelo, enquanto que o desempenho gerencial (DG) explica 20,2\%, 0 empowerment psicológico (EP) explica 35,5\%, o uso diagnostico (UD) explica 35,2\% e o uso interativo (UI) explica $21,4 \%$ do modelo proposto. Marginson et al. (2014) encontraram em seu estudo um R2 de 0,143 para AP, de 0,311 para EP, de 0,269 para DG, de 0,214 para UD e de 0,184 para UI. Denota-se que os valores encontrados em ambos estudos se assemelham em relação ao valor explicativo da variável endógena.

\section{Discussão dos resultados}

Nesta seção procede-se à análise das hipóteses formuladas no estudo de Marginson et al. (2014), e que orientaram esta pesquisa. Na H1, observa-se associação positiva e significativa entre uso diagnóstico de medidas de avaliação de desempenho e ambiguidade de papéis. Assim, rejeita-se a H1. Porém, a associação entre uso diagnóstico e 
empowerment psicológico não se apresenta significativa. Esses resultados divergem dos observados por Marginson et al. (2014), em que as medidas de avaliação de desempenho, quer de uso diagnóstico e interativo, foram fontes eficazes de informação para neutralizar a ambiguidade de papéis dos gestores, assegurando maior comprometimento com a organização. Duas situações podem ter contribuído para esses resultados.

A primeira, relacionada ao uso diagnóstico com ambiguidade de papéis (H1 sig.), na cooperativa pesquisada, os respondentes indicaram ambiguidade de papéis, conforme Tabela 2 (média de 6,04). No entanto, Henri (2006) alerta que o uso diagnóstico leva a impacto negativo, devido ao estresse com as consequências negativas do controle diagnóstico. A segunda situação, relacionada ao uso diagnóstico com empowerment psicológico (H1 não sig.), na cooperativa pesquisada, o sistema de controle diagnóstico não influenciou positiva ou negativamente o empowerment psicológico. Decorre que o empowerment é um construto multidimensional, que suporta a eficácia organizacional e o desempenho pela delegação de autoridade e construção motivacional (Conger e Kanungo, 1988; Hall, 2008).

$\mathrm{Na} \mathrm{H2}$, a associação do uso interativo com o empowerment psicológico não foi significativa, assim, rejeita-se a H2. A associação do uso interativo com ambiguidade de papéis também não foi significativa. Portanto, o uso do sistema de controle interativo não influencia positiva e nem negativamente o empowerment psicológico e a ambiguidade de papéis, indicando que os respondentes não observam controle interativo entre as gerências, ao contrário do que foi constatado no estudo de Marginson et al. (2014). Conforme a literatura, o uso interativo de medidas de avaliação de desempenho pode reduzir a ambiguidade de papéis e aumentar o empowerment psicológico, incentivando o debate entre os gestores e levar a um acordo sobre como lidar com incertezas (Daft e Lengel, 1990).

Comunicação e feedback mais efetivos entre os gestores nesta organização ajudaria a reduzir a ambiguidade de papéis e aumentar o empowerment psicológico. Adicionalmente, a literatura indica que ambos sistemas de controle, diagnóstico e interativo, impactam no desenvolvimento e revisão de metas (Simons, 1995; Simons, 2005) e são suscetíveis de encorajar e motivar os indivíduos, o que não foi constado na organização pesquisada. No sistema de controle interativo, propõe-se que as metas sejam esclarecidas pelo incentivo de participação dos subordinados e pelas interações interpessoais (Simons, 1995; Simons, 2005), aspecto também não observado nesta pesquisa. 
$\mathrm{Na}$ H3, as variáveis ambiguidade de papéis e empowerment psicológico apresentam associação negativa e não significativa. Infere-se que a ambiguidade de papéis não afeta 0 empowerment psicológico na organização estudada, assim rejeita-se a H3. Esse resultado diverge do estudo de Marginson et al. (2014), que constatou relação significativa entre essas variáveis. Isso pressupõe uma construção relacional de poder ou motivacional, não observada na cooperativa investigada. Marginson e Ogden (2005) argumentam a partir de um conceito relacional de poder, que a autoridade é difundida em toda a organização, que as regras fluem e as comunicações laterais predominam sobre a hierarquia.

$\mathrm{Na} \mathrm{H} 4 \mathrm{a}$, a associação entre ambiguidade de papéis e desempenho gerencial apresentou-se negativa e significativa, assim não rejeita-se a $\mathrm{H} 4 \mathrm{a}$. Esse resultado coaduna com o de Marginson et al. (2014). Isso implica que a falta de informações sobre expectativas e metas de papéis pode resultar em esforço ineficiente, mal orientado e/ou insuficiente para o desempenho das atividades, o que, consequentemente, reduz o desempenho (Hall, 2008).

$\mathrm{Na}$ H4b ocorreu associação positiva e significativa entre empowerment psicológico e desempenho gerencial, portanto não rejeita-se a $\mathrm{H} 4 \mathrm{~b}$. Esses resultados coadunam com os de Marginson et al. (2014). Depreende-se que a intensidade do esforço, o tempo do esforço e a flexibilidade dos gestores podem influenciar na eficácia. 0 desempenho no trabalho aumenta se os gestores percebem autonomia na realização do seu trabalho (Miller e Monge, 1986). Pessoas que se sentem competentes tendem a serem mais propensas a agir com competência (Gist e Mitchell, 1992). Indivíduos que atribuem mais significado ao seu trabalho se esforçam mais, são mais comprometidos com suas tarefas (Hall, 2008).

Finalmente, os resultados sugerem que as medidas de avaliação de desempenho estão indiretamente relacionadas com o desempenho gerencial pelos efeitos nos caminhos de controle entre o sistema de controle diagnóstico (significativo), contudo, para o sistema de controle interativo (não significativo) nenhum efeito direto é observado. No estudo de Marginson et al. (2014) isso foi abordado de forma exploratória, já que não foram apresentados argumentos teóricos. No entanto, o modelo conceitual apresenta como hipótese geral a mediação dos efeitos psicológicos (ambiguidade de papéis e empowerment psicológico), na relação entre os sistemas de controle gerencial (diagnóstico e interativo das medidas de avaliação de desempenho) e o desempenho gerencial. 


\section{Considerações finais}

Este estudo objetivou verificar a associação do uso diagnóstico e interativo de medidas de avaliação de desempenho com a diminuição da ambiguidade de papéis e o aumento de empoderamento psicológico de gestores. Esta pesquisa, realizada em uma grande cooperativa agroindustrial, com diversas unidades estabelecidas na regiões Sul e Centro-0este do Brasil, foi inspirada no estudo de Marginson et al. (2014), realizado com gestores da NewCom, maior empresa mundial do setor de telecomunicações da Nova Zelândia. É esperado que princípios organizacionais distintos, como no caso de uma cooperativa, tenham efeitos psicológicos diferentes nas medidas de avaliação de desempenho.

A análise descritiva evidencia altos índices de ambiguidade de papéis, indicando que não há clareza nas informações e especificações de execução das tarefas dos gestores. Quanto ao empoderamento psicológico, os resultados mostram que os gestores possuem capacidade de realizar atividades específicas com habilidade e que o impacto psicológico pode influenciar o resultado dos gestores no trabalho. Observou-se também que há um elevado nível de envolvimento dos gestores no desempenho gerencial e nas medidas de avaliação de desempenho. Isso sugere que os gestores possuem comprometimento com os sistemas de controle concebidos para assegurar a realização das metas.

Na modelagem de equações estruturais, foi observado na $H 1$, que uso diagnóstico de medidas de avaliação de desempenho está associado positiva e significativamente com ambiguidade de papéis. Isso indica que na cooperativa pesquisada há dissonâncias no cumprimento das metas em relação aos papéis desempenhados pelos gestores. Especula-se que, no caso, os princípios cooperativos se sobrepõem à clareza de papéis no cumprimento das metas pelos gestores. Este resultado difere do estudo de Marginson et al. (2014), já que nele, as medidas de avaliação de desempenho se mostraram fontes eficazes de informação para neutralizar a ambiguidade de papéis dos gestores, assegurando maior comprometimento com a organização.

$\mathrm{Na} \mathrm{H} 2$, a associação do uso interativo com o empowerment psicológico não foi significativa, da mesma forma como a associação do uso interativo com ambiguidade de papéis. Depreende-se que o sistema de avaliação de desempenho e os problemas inesperados não afetam a capacitação psicológica e a confiança e engajamento dos gestores. Tais resultados não corroboram os do estudo base. $\mathrm{Na} \mathrm{H} 3$, ambiguidade de papéis e empowerment psicológico apresentaram associação negativa não significativa. Infere-se que a ambiguidade de papéis não afeta o empowerment psicológico na organização 
estudada, indicando que a autoridade exercida pelos gestores flui e o nível hierárquico predomina. Isso diverge do estudo base, talvez pelos efeitos psicológicos distintos da avaliação de desempenho.

$\mathrm{Na} \mathrm{H} 4 \mathrm{a}$, ambiguidade de papéis e desempenho gerencial demonstrou associação negativa e significativa. Isso implica menor desempenho dos gestores pela falta de informações sobre expectativas de papéis, resultando em esforço ineficiente, mal orientado e/ou insuficiente para o desempenho das atividades. Observou-se também que os efeitos indiretos do sistema de controle influenciam na ambiguidade de papéis e, consequentemente, no desempenho gerencial. Na H4b, o empowerment psicológico mostrou-se associado positiva e significativamente com desempenho gerencial. Corrobora-se assim em ambas as hipóteses os achados do estudo de Marginson et al. (2014).

De modo geral, os resultados desta pesquisa não se apresentaram similares aos do estudo base, uma vez que as três hipóteses centrais, isto é, as relacionadas aos efeitos psicológicos dos sistemas de controle se mostraram contrários. Tal pode ser motivado pelo fato da pesquisa ter sido replicada em uma organização com diferente constituição jurídica (organização cooperativa), pertencente a outro setor econômico e inserida em um ambiente social e econômico distinto. Portanto, observa-se uma lacuna de pesquisa a ser explorada, isto é, em que medida as diferenças nos princípios organizacionais, no caso de uma cooperativa, explicam os efeitos psicológicos distintos nas medidas de avaliação de desempenho.

Além disso, os resultados desta pesquisa não apoiam a posição de que as medidas de avaliação de desempenho, quer de uso diagnóstico ou interativo, são fontes eficazes de informação para neutralizar a ambiguidade de papéis. Já no estudo base ficou evidenciado que, pela clareza do papel e do controle diagnóstico pode-se reduzir os níveis de ambiguidade, estabelecendo metas claras e oferecer suporte à aprendizagem por meio de um único feedback. Por outro lado, ao promover o debate, o uso interativo das medidas de avaliação de desempenho ajuda a reduzir a ambiguidade de papéis.

Conclui-se que o uso diagnóstico de medidas de avaliação de desempenho está associado com ambiguidade de papéis, ao contrário do uso interativo, que não está associado. Além disso, os sistemas de controle das medidas de avaliação de desempenho não afetam a capacitação psicológica e o engajamento dos gestores. Da mesma forma, a ambiguidade de papéis não afeta o empoderamento psicológico, pois a autoridade dos gestores flui e o nível hierárquico predomina. Tal sugere que o desenho institucional da cooperativa possa 
ter influído nos efeitos psicológicos analisados. No entanto, o desempenho gerencial dos gestores é afetado pela falta de informações sobre expectativas e metas de papéis.

É importante destacar que os resultados obtidos devem ser tomados com cautela em função das implicações do número reduzido de respondentes e da pesquisa se restringir ao ambiente de uma só organização. Contudo, as evidências obtidas na modelagem de equações estruturais contribuem para avanços teórico-empíricos da temática. Destaca-se em especial o fato do estudo discorrer sobre os impactos psicológicos dos sistemas de controle diagnóstico e interativo nas medidas de avaliação de desempenho. Além disso, a pesquisa abre a possibilidade de realizar estudos comparativos, já que foi realizada em uma organização sob a orientação de princípios cooperativos, distinta da empresa mundial do setor de telecomunicações investigada por Marginson et al. (2014).

Ressalta-se que não foram utilizados os caminhos de controle expostos com ponto de interrogação no modelo teórico, devido ao fato de não terem respaldo teórico suficiente, sendo que no artigo base o autor abordou-os de forma exploratória. Assim, recomenda-se para pesquisas futuras a investigação desses caminhos de controle em organizações similares ou diferentes. Além disso, como o estudo foi realizado no segmento de cooperativas e em apenas uma organização, os resultados não podem ser generalizados e estendidos para outras organizações. Assim recomenda-se a reaplicação da pesquisa em diversos tipos de organização para cotejar os resultados.

\section{Referências}

Anthony, R., \& Govindarajan, V. (2008). Sistema de controle gerencial. São Paulo: McGraw Hill. Bido, D., Silva, D., Souza, C., \& Godoy, A. (2010). Mensuração com indicadores formativos nas pesquisas em administração de empresas: como lidar com multicolinearidade entre eles? Administração: Ensino e Pesquisa, 11(2), 45-269.

Chiles, A., \& Zorn, T. (1995). Empowerment in organizations: employees' perceptions of the influences of empowerment. Journal of Applied Communication Research, 23(1), 1-25.

Chin, W. (1998). The partial least squares approach for structural equation modeling. In Marcoulides, G. (ed.), Modern methods for business research (pp. 295-336). London: Lawrence Erlbaum Associates.

Churchill Jr., G., Ford, N., \& Walker Jr., 0. (1976). Organizational climate and job satisfaction in the sales force. Journal of Marketing Research, 13(1), 323-332.

Clark, R., Hartline, M., \& Jones, K. (2009). The effects of leadership style on hotel employees commitment to service quality. Cornell Hospitality Quarterly, 50(2), 209-230. 
Cohen, J. (1988). Statistical power analysis for the behavioral sciences, 2nd ed. Hillsdale, NJ: Lawrence Erlbaum Associates.

Collins, F. (1982). Managerial accounting systems and organizational control: A role perspective. Accounting, Organizations and Society, 7(2), 107-112.

Conger, J., \& Kanungo, R. (1988). The empowerment process: integrating theory and practice. Academy of Management Review, 13(3), 471-482.

Daft, R., \& Lengel, R. (1990). Information richness: A new approach to managerial behavior and organizational design. In Cummings, L., \& Staw, B. (eds.), Information and cognition in organizations (pp. 191-233). Greenwich: Jai Press.

Davison, A., \& Hinkley, D. (2003). Bootstrap methods and their application, 2nd ed. New York: Cambridge University Press.

Ford, R., \& Fottler, M. (1995). Empowerment: A matter of degree. Academy of Management Executive, 9(3), 21-28.

Fornell, C., \& Larcker, D. (1981). Evaluating structural equation models with unobservable variables and measurement error. Journal of Marketing Research, 18(1), 39-50.

Gist, M., \& Mitchell, T. (1992). Self-efficacy: a theoretical analysis of its determinants and malleability. Academy of Management Review, 17(2), 183-211.

Gomes, C., Yasin, M., \& Lisboa, J. (2004). A literature review of manufacturing performance measures and measurement in an organizational context: A framework and direction for future research. Journal of Manufacturing Technology Management, 15(6), 511-530.

Gunasekaran, A., Patel, C., \& Mcgaughey, R. (2004). A framework for supply chain performance measurement. International Journal of Production Economics, 87(3), 333-347.

Hair Jr., J., Black, W., Babin, B., Anderson, R., \& Tatham, R. (2009). Análise multivariada de dados, 6 ed. Porto Alegre: Bookman.

Hall, M. (2008). The effect of comprehensive performance measurement systems on role clarity, psychological empowerment and managerial performance. Accounting, Organizations and Society, 33(2/3), 141-163.

Hechanova, R., Alampay, R., \& Franco, E. (2009). Psychological empowerment, job satisfaction, and performance among Filipino service workers. Asian Journal of Social Psychology, 9(1), $72-78$.

Henri, J. (2006). Management control systems and strategy: a resource based perspective. Accounting, Organizations and Society, 31(6), 529-558.

Henseler, J., Ringle, C., \& Sinkovics, R. (2009). The use of partial least squares path modeling in international marketing. Advances in International Marketing, 20(1), 277-319.

House, R., \& Rizzo, J. (1972). Role conflict and role ambiguity as critical variables in a model of organizational behavior. Academy of Management Journal, 25(2), 452-456. 
Hulland, J. (1999). Use of partial least squares (PLS) in strategic management research: a review of four recent studies. Strategic Management Journal, 20(2), 195-204.

Kahn, R. (1973). Conflict, ambiguity, and overload: three elements in job stress. In MaClean, A. (ed.), Occupational stress (pp. 47-61). Springfield: Gulf Publisher.

Kahn, R., Wolfe, D., Quinn, R., Snoek, J., \& Rosenthal, R. (1964). Organizational stress: Studies in role conflict and ambiguity. New York: Jonh Wiley.

Katz, D., \& Kahn, R. (1978). The social psychology of organizations. New York: John Wiley.

Klann, R., \&Beuren, I. (2014). Relações do empowerment psicológico com o sistema de mensuração de desempenho e o desempenho gerencial. Revista Ambiente Contábil, 6(3), 116-133.

Konczak, L., Stelly, D., \& Trusty, M. (2000). Defining and measuring empowering leader behaviors: development of an upward feedback instrument. Educational and Psychological Measurement, 60(2), 301-312.

Liden, R., Wayne, S., \& Sparrowe, R. (2000). An examination of the mediating role of psychological empowerment on the relations between the job, interpersonal relationships, and work outcome. Journal of Applied Psychology, 85(3), 407-416.

Lima, E., Costa, S., \& Angelis, J. (2008). The strategic management of operations system performance. International Journal of Business Performance Management, 10(1), 108-132.

Marginson, D., Mcaulay, L., Roush, M., \& Van Zijl, T. (2014). Examining a positive psychological role for performance measures. Management Accounting Research, 25(1), 63-75

McNally, G. (1980). Responsibility accounting and organizational control: Some perspectives and prospects. Journal of Business Finance \& Accounting, 7(2), 165-181.

Michaels, R., Ralph L., \& Erich A. (1987). Role stress among industrial buyers: An integrative model. Journal of Marketing, 51(1), 28-45.

Miller, K., \& Monge, P. (1986). Participation, satisfaction, and productivity: A meta-analytic review. Academy of Management Journal, 29(4), 727-753.

Nascimento, S., \&Beuren, I. (2014). Impacto do sistema de recompensa e do acesso às informações sobre o desempenho individual no empowerment psicológico e o seu reflexo na eficácia gerencial de empresa multinacional. Sociedade, Contabilidade e Gestão, 9(1), 6-24.

Neely, A. (2005). The evolution of performance measurement research: developments in the last decade and a research agenda for the next. International Journal of Operations and Production Management, 25(12), 1264-1277.

Neely, A., Mills, J., Gregory, M., \& Platts, K. (1995). Performance measurement system desing: a literaure review and research agenda. International Journal of Operations \& Production Management, 15(4), 80-116.

Quinn, R., \& Spreitzer, G. (1997). The road to empowerment: Seven questions every leader should consider. Organizational Dynamics, 26(2), 37-49. 
Rizzo, J., House, R., \& Lirtzman, S. (1970). Role conflict and ambiguity in complex organizations. Administrative Science Quarterly, 15(2), 150-163.

Seibert, S., Wang, G., \& Courtright, S. (2011). Antecedents and consequences of psychological and team empowerment in organizations: a meta-analytic review. Journal of Applied Psychology, 96(5), 981-1003.

Simons, R. (1995). Levers of control: how managers use innovative control systems to drive strategic renewal. Boston: Harvard Business School.

Simons, R. (2005). Levers of organizational design: How managers use accountability systems for greater performance and commitment. Boston: Harvard Business School.

Spreitzer, G. (1995). Psychological empowerment in the workplace: Dimensions, measurement, and validation. Academy of Management Journal, 38(5), 1442-1466.

Tan, K., \& Platts, K. (2009). Linking operations objectives to actions: a plug and play approach. International Journal of Production Economics, 121(2), 610-619.

Thomas, K., \& Velthouse, B. (1990). Cognitive elements of empowerment: an interpretive model of intrinsic task motivation. Academy of Management Review, 15(4), 666-681. 